\title{
Promoter effects of adeno-associated viral vector for transgene expression in the cochlea in vivo
}

\author{
Yuhe $\mathrm{Liu}^{1,3}$, Takashi Okada ${ }^{2}$, \\ Tatsuya Nomoto ${ }^{2}$, Xiaomei $\mathrm{Ke}^{1}$, \\ Akihiro Kume ${ }^{2}$, Keiya Ozawa ${ }^{2}$ \\ and Shuifang Xiao ${ }^{1}$ \\ ${ }^{1}$ Department of Otolaryngology \\ Head and Neck Surgery, Peking University First Hospital \\ Beijing 100034, China \\ ${ }^{2}$ Division of Genetic Therapeutics \\ Center for Molecular Medicine \\ Jichi Medical School \\ Tochigi 329-0498, Japan \\ ${ }^{3}$ Corresponding author: Tel, 86-10-63078547; \\ Fax, 86-10-66173427; E-mail, liuyuhe@xinhuanet.com
}

Accepted 6 February 2007

Abbreviations: AAV, adeno-associated virus; CAG, cytomegalovirus IE enhancer and chicken $\beta$-actin promoter; CMV, cytomegalovirus promoter; EF-1 $\alpha$, elongation factor 1 alpha promoter; EGFP, enhanced green fluorescent protein; ITRs, inverted terminal repeats; Myo, myosin 7A promoter; NSE, neuron-specific enolase promoter; RSV, Rous sarcoma virus promoter; WPRE, woodchuck hepatitis; virus posttranscriptional regulatory element

\begin{abstract}
The aims of this study were to evaluate the expression of enhanced green fluorescent protein (EGFP) driven by 6 different promoters, including cytomegalovirus IE enhancer and chicken $\beta$-actin promoter (CAG), cytomegalovirus promoter (CMV), neuron-specific enolase promoter (NSE), myosin 7A promoter (Myo), elongation factor $1 \alpha$ promoter (EF-1 $\alpha$ ), and Rous sarcoma virus promoter (RSV), and assess the dose response of CAG promoter to transgene expression in the cochlea. Serotype 1 adeno-associated virus (AAV1) vectors with various constructs were transduced into the cochleae, and the level of EGFP expression was examined. We found the highest EGFP expression in the inner hair cells and other cochlear cells when CAG promoter was used. The CMV and NSE promoter drove the higher EGFP expression, but only a marginal activity was ob served in EF-1 $\alpha$ promoter driven constructs. RSV promoter failed to drive the EGFP expression. Myo promoter driven EGFP was exclusively expressed in
\end{abstract}

the inner hair cells of the cochlea. When driven by CAG promoter, reporter gene expression was detected in inner hair cells at a dose as low as $3 \times 10^{7}$ genome copies, and continued to increase in a dosedependent manner. Our data showed that individual promoter has different ability to drive reporter gene expression in the cochlear cells. Our results might provide important information with regard to the role of promoters in regulating transgene expression and for the proper design of vectors for gene expression and gene therapy.

Keywords: cochlea; dependovirus; gene therapy; gene transfer techniques; green fluorescent proteins; promoter regions

\section{Introduction}

Gene transfer is a promising tool to study the physiology of the cochlea and cochlear cells. The feasibility of gene therapy in the cochlea has been established (Dazert et al., 2001; Kawamoto et al., 2001; Liu et al., 2005), still, there is a controversy about the transduction of the hair cells or spiral ganglion cell, even when the same vehicle is employed as vectors, such as adenovirus and adenoassociated virus (AAV) (Luebke et al., 2001; Li et al., 2002; Yamasoba et al., 2002; Liu et al., 2005). The previous studies support the hypothesis that this discrepancy may be caused by the differences in delivery methods or driven promoters. It has been shown that individual promoter has distinct ability to express reporter genes in different cell types (Chung et al., 2002; Nomoto et al., 2003; Shevtsova et al., 2005). Tissue or cell specific promoters are capable of restricting gene expression in desirable cells and facilitating persistent or regulated transgene expression (Liu et al., 2004). The cytomegalovirus IE enhancer and chicken $\beta$-actin promoter (CAG) drives high level of transgene expression and is one of the most commonly used promoters for gene transfer (Xu et al., 2001). The myosin 7A promoter (Myo) was shown to be the specific promoter of hair cells in the cochlea (Boeda et al., 2001). We have previously demonstrated that, with AAV1 vectors, the CAG promoter can drive transgene expression in the cochlea cells at a highly functional level (Liu et al., 2005). In the present study, we systematically evaluated the promoters CAG, Myo, cytomegalovirus 
promoter (CMV), elongation factor 1 alpha promoter $(E F-1 \alpha)$, neuron-specific enolase promoter (NSE), and rous sarcoma virus promoter (RSV) for their abilities in gene transfers to the cochlear cells using in vivo assays. We also examined the dose-response relationship for CAG promoter over a broad range in the cochlea.

\section{Materials and Methods}

\section{Construction and preparation of the proviral plasmids}

The AAV1 vector proviral plasmid, pAAV2-LacZ, harbors an Escherichia coli $\beta$-galactosidase expression cassette with the CMV promoter, human growth hormone first intron, and SV40 early polyadenylation sequence; flanked by inverted terminal repeats (ITRs) (Okada et al., 2002). The proviral plasmid pAAV2-CAG-EGFP-WPRE (pAAV2-CAG) consists of enhanced green fluorescent protein (EGFP) gene under the control of the CAG promoter and Woodchuck hepatitis virus posttranscriptional regulatory element (WPRE) and is flanked by ITRs (Liu et al., 2005). Agel restriction site was created on 5 ' end of Myo promoter (Boeda et al., 2001) from C2C12 cell line genomic DNA when subcloned into pCRIITOPO by PCR (forward primer: 5'-ATGTCGACCTTGGGCAACCTCTAGACG-3'; reverse primer: 5'-ATCCGCGGCTTCTACGTCTGCACAC-3'). Spel-Agel fragment containing the Myo promoter was subcloned into pAAV2-CAG to obtain pAAV2-Myo-EGFP-WPRE (pAAV2-Myo). PAAV2-CMV-EGFP-WPRE (pAAV2CMV), pAAV2-EF1 $\alpha$-EGFP-WPRE (pAAV2-EF1 $\alpha$ ), pAAV2-NSE-EGFP-WPRE (pAAV2-NSE), and pAAV2RSV-EGFP-WPRE (pAAV2-RSV) were constructed as previously described (Ogasawara et al., 1998; Nomoto et al., 2003; Mochizuki et al., 2004). A schematic illustration of the AAV1 vectors is shown in Figure 1. AAV1-helper plasmid harbors Rep and Cap. Adenovirus helper plasmid pAdeno5 identical to pVAE2AE4-5 encodes the entire E2A and E4 regions, and VA RNA I and II genes (Matsushita et

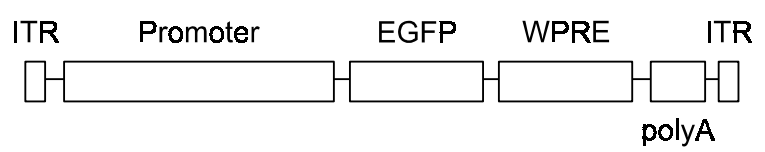

Figure1. Schematic representations of viral vectors constructed. AAV1 vectors were constructed using different promoters to drive reporter gene EGFP and the SV40 polyadenylation sequences (polyA). The promoters in the study include CAG, CMV, NSE, Myo, EF-1 $\alpha$ and RSV. The Woodchuck hepatitis virus posttranscriptional regulatory element (WPRE) was inserted $3^{\prime}$ of the EGFP cassette in the vectors. ITR, inverted terminal repeats. al., 1998). Plasmids were purified with the QIAGEN plasmid purification Kits (QIAGEN K.K., Tokyo, Japan).

\section{Recombinant AAV1 vectors production}

Vectors were produced with the AAV1 packaging plasmids $p A A V 1$ RepCap and the AAV1 proviral plasmid pAAV2-CAG, pAAV2-Myo, pAAV2-CMV, pAAV2EF1 $\alpha$, pAAV2-NSE, or pAAV2-RSV. Six kinds of AAV1 vectors were produced using three-plasmid transfection adenovirus-free protocol (Liu et al., 2005). Recombinant AAV1 was harvested at $72 \mathrm{~h}$ after transfection by three cycles of freeze/thawing. The crude viral lysate was then purified twice on a cesium chloride $(\mathrm{CsCl})$ two-tier centrifugation gradient as previously described (Okada et al., 2002). The viral stock was titrated by the quantitative realtime PCR of DNase-treated stocks with plasmid standards (Veldwijk et al., 2002). Thus, AAV1 vectors of AAV1-CAG-EGFP-WPRE (AAV1-CAG), AAV1-MyoEGFP-WPRE (AAV1-Myo), AAV1-CMV-EGFP-WPRE (AAV1-CMV), AAV1-EF1 $\alpha$-EGFP-WPRE (AAV1EF1 $\alpha$ ), AAV1-NSE-EGFP-WPRE (AAV1-NSE), and AAV1-RSV-EGFP-WPRE (AAV1-RSV) were constructed. The titers of AAV1 vectors were from $6 \times$ $10^{12}$ to $8 \times 10^{13}$ genome copies (g.c.). For doseresponse relationship experiment, vector stocks were serially diluted with artificial perilymph (AP: 145 $\mathrm{mM} \mathrm{NaCl}, 2.7 \mathrm{mM} \mathrm{KCl}, 2 \mathrm{mM} \mathrm{MgSO}_{4}, 1.2 \mathrm{mM} \mathrm{CaCl}_{2}$, 5 mM HEPES).

\section{Evaluation of the in vitro activity of recombinant AAV1 vectors}

To examine the functions of individual promoters, HEK 293 cells were cultured for $36 \mathrm{~h}$, and then transfected with various AAV1 vectors separately $(1 \times$ $10^{4}$ g.c./cell). Myosin $7 A$ gene is transcribed in several epithelial cell types that possess microvilli, i.e. renal epithelial cell. Since HEK 293 cell line has been originated from human embryonic kidney epithelial cell, the Myo promoter activity can be assessed in this particular cell line. Forty-eight $h$ after transduction, the cells were recorded for EGFP expression using OLYMPUS IX70 (Olympus corporation, Tokyo, Japan) fluorescence microscope. Cells with green fluorescence were considered "positive" for transgene expression.

\section{Surgical procedures and cochlear perfusions}

All animal studies were approved by the ethics committee of Jichi Medical School in Japan and Peking University First Hospital in China. They were performed following the animal research guidelines at Jichi Medical School and Peking University First 
Hospital. Seventy female C57BL/6J mice (4 weeks old, CLEA Japan, Tokyo, Japan) and 30 male Sprague-Dawley rats (5 weeks old, CLEA Japan, Tokyo, Japan) with normal Preyer's reflexes were included in this study. Surgical procedures and cochlear perfusion of the animals were performed as previously described (Liu et al., 2005). In the testing groups, 5 $\mu \mathrm{l}$ of AAV1 vectors solution was microinjected into the unilateral cochlea. Five mice received control cochlear perfusions with artificial perilymph only. Each AAV1 vector was injected into 5 animals with 3 $\times 10^{10}$ g.c./cochlea. Injected dose of AAV1-CAG vector into 5 mice was varied from $3 \times 10^{10}$ to $3 \times$ $10^{7}$ g.c./cochlea for dose response study.

\section{Histological study}

The capability of the transgene expression in the cochlear cells for various AAV1 vectors was determined by visualizing EGFP levels. The animals were sacrificed 2 weeks after injection, and the cochleae were harvested and the stapes footplates were removed. After fixing and decalcifying, the cochleae were made by cryosection $(10 \mu \mathrm{m})$. The EGFP expression was detected under OLYMPUS IX70 fluorescence microscope using a standard fluoresce in isothiocyanate (FITC) filter set and a Studio Lite software (Olympus corporation, Tokyo, Japan). Level of expression was graded by fluorescent intensity.

\section{Results}

\section{Evaluation of activity of all promoters in vitro}

The EGFP expression in the HEK 293 cell was detected with any of the AAV1 vectors, indicating that all promoters were functional. However, their expression levels were different (Figure 2). Robust EGFP expression with CAG promoter was shown in the HEK 293 cell, followed by CMV, NSE, EF-1 $\alpha$ and RSV. Myo promoter was just weak in 293 cells.

\section{EGFP expression profile within the cochlea under different promoter}

The pattern of EGFP expression in the cochlear cells was quite similar in both mice and rat for each promoter. Distribution of AAV1 vector-mediated EGFP expression was examined throughout the cochlea for all promoters tested (Table 1). With CAG promoter, a robust EGFP expression was identified in the various cochlear cells, including the inner hair cells, spiral ganglion cells, inner sulcus cells, Hensen's cells and mesenchymal cells (Figure 3B). A

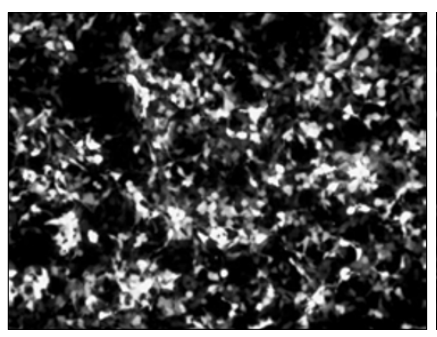

AAV1-CAG

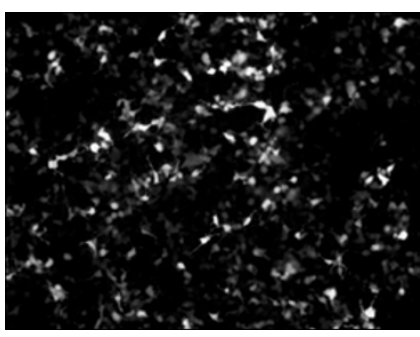

AAV1-NSE

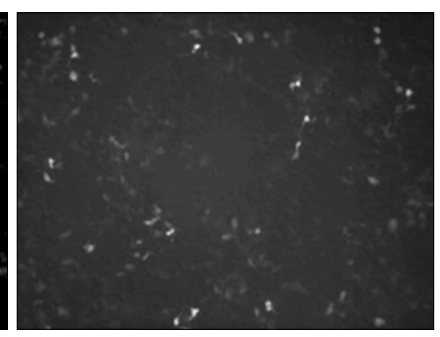

AAV1-Myo
Figure 2. EGFP expression in the HEK293 cell transduced with AAV1 vectors harboring distinct promoters. Robust EGFP expression with CAG promoter was shown, EGFP expression with CMV is similar to that with CAG, and EGFP expression with NSE to with RSV and EF-1 $\alpha$ Myo promoter was just weak in 293 cells.

Table 1. Transgene expression with distinct promoter in the cochlear cells

\begin{tabular}{|c|c|c|c|c|c|c|c|c|c|}
\hline Promoter & $\begin{array}{l}\text { Inner hair } \\
\text { cells }\end{array}$ & $\begin{array}{l}\text { Outer hair } \\
\text { cells }\end{array}$ & $\begin{array}{c}\text { Spiral } \\
\text { ganglion } \\
\text { cells }\end{array}$ & $\begin{array}{l}\text { Stria vascularis } \\
\text { cells }\end{array}$ & $\begin{array}{l}\text { Spiral ligament } \\
\text { cells }\end{array}$ & $\begin{array}{l}\text { Reissner's } \\
\text { membrane }\end{array}$ & $\begin{array}{l}\text { Inner sulcus } \\
\text { cells }\end{array}$ & Claudius' cells & $\begin{array}{c}\text { Mesenchymal } \\
\text { cells }\end{array}$ \\
\hline CAG & + & - & + & - & + & + & + & - & + \\
\hline CMV & + & - & + & - & + & + & + & - & + \\
\hline NSE & - & - & + & - & + & + & - & - & + \\
\hline $\mathrm{EF}-1 \alpha$ & - & - & + & - & + & - & - & - & + \\
\hline Myo & + & - & - & - & - & - & - & - & - \\
\hline RSV & - & - & - & - & - & - & - & - & - \\
\hline
\end{tabular}

+ means EGFP expression in the cells, while - means no fluorescence in the cells. 


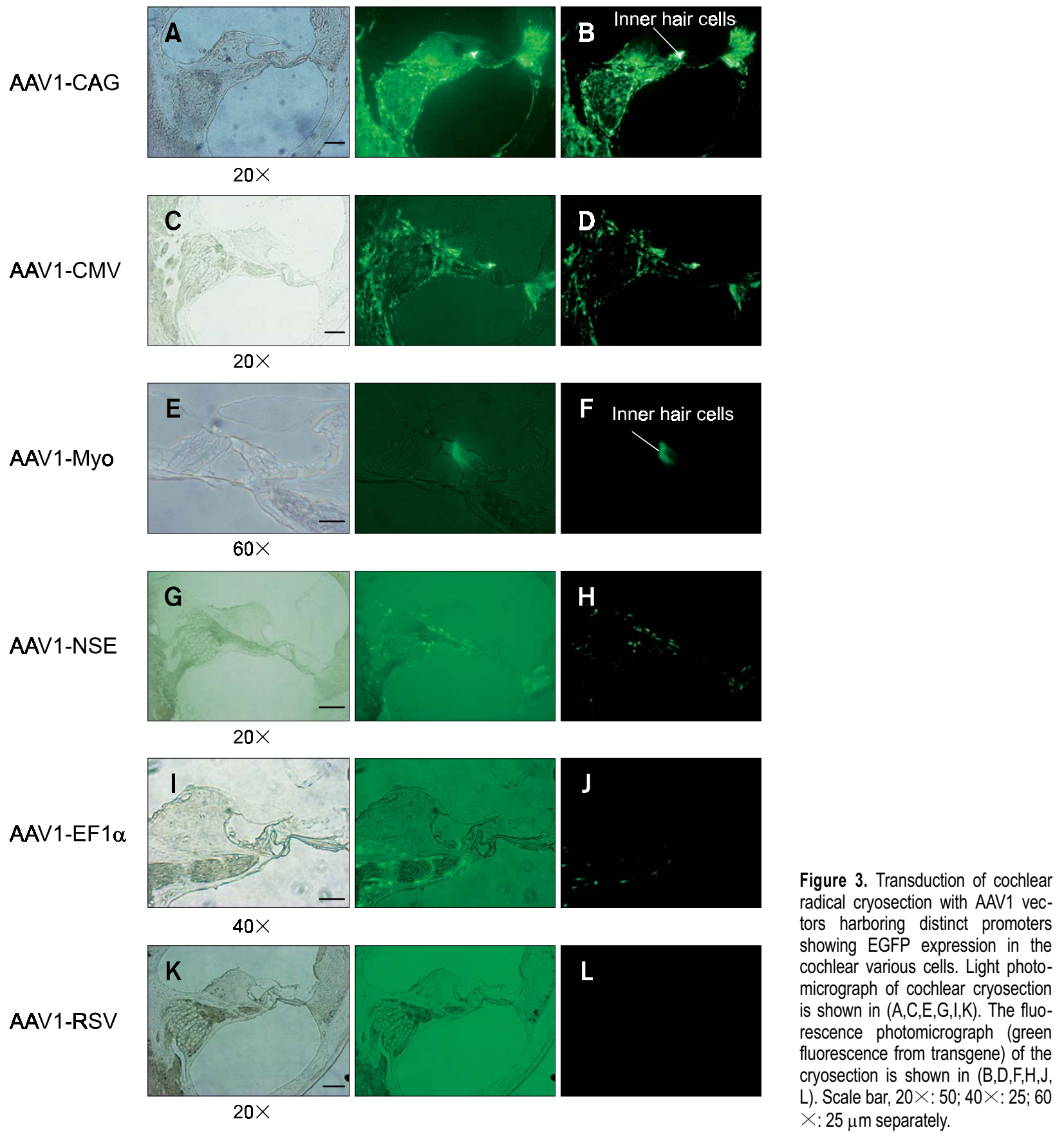

similar transgene expression pattern (Figure 3D) was also observed when AAV1-CMV vector was used. In contrast, the EGFP expression under NSE and EF- $1 \alpha$ promoters was present in all kinds but the inner hair cells of cochlear cells (Figure $3 \mathrm{H}$ and J). As reported previously (Boeda et al., 2001), Myo promoter driven EGFP was exclusively expressed in the inner hair cells of cochlea (Figure 3F), while EGFP expression in the cochlear cells was rarely observed with the RSV promoter (Figure 3L). Consistent with the previous findings (Liu et al., 2005), no EGFP expression was detected in the outer hair cells, supporting pillar cells, or the stria vascularis cells with all tested promoters. The level of EGFP expression in the cochlear cells was highest with CAG promoter, followed by CMV promoter. NSE promoter only drove weak expression of EGFP, while EF-1 $\alpha$ promoter displayed the least activity. 
Our results indicate that, among these promoters, CAG promoter was the most efficient in transducing the cochlear cells.

\section{Dose-response relationship for AAV1-CAG vector}

Within the dose-effect groups with AAV1-CAG, a significant effect of dosage on the number of EGFPexpressing cochlear cells was found by one-way ANOVA $(P<0.001)$. CAG promoter driven EGFP expression in inner hair cells was detected at a dose as low as $3 \times 10^{7}$ genome copies, with expression increasing in a dose-dependent manner.

\section{Discussion}

The various factors affecting the transcription of a transgene includes the promoter containing 5 'flanking region, 3'UTR, enhancer, suppressor, insulator sequences and the type and activity of transcription factors available in the transfected cells. This study showed that all 6 promoters, including viral, mammalian cells promoters, were capable of driving EGFP expression in HEK 293 cells, suggesting that the HEK 293 cell possesses all essential transcription factors for recognizing the diverse promoter sequences applied in this study. The CAG promoter appeared to be more efficient than other promoters based on the following observations: (1) more cells transduced in an equal dose comparison; (2) greater spreads of EGFP-expressing cell groups; and (3) CAG promoter-driven expression in more cell types, especially the inner hair cells, which were rarely found with other promoters except for CMV promoter. These results extend our knowledge about the promoter-related characteristics of AAV1-mediated gene transfer in the cochlea.

Interestingly, our data demonstrate that, in comparison with NSE, EF- $1 \alpha$ and RSV promoters, CAG, CMV and hair cells specific promoter-Myo can drive EGFP expression in the inner hair cells. Even though certain promoters, such as EF-1 $\alpha$ and RSV promoters, showed robust activity in many tissues, their activities are limited in the cochlear cells. Nowadays, it still has not been known why NSE, EF-1 $\alpha$ and RSV promoters are inactive in the inner hair cells. One likely explanation is that inner hair cells may not express some transcription factors, which were necessary for these promoters to be actived. On the other hand, the inner hair cells may have all necessary components for the full transcriptional activity of the CAG, CMV or Myo promoters. Another possibility is transcriptional shutdown or promoter silencing in the inner hair cells. These phenomena have been observed previously in different organs such as the liver, lungs, and muscles (Hartikka et al.,
1996; Chen et al., 2001; Gill et al., 2001). Besides the known mechanisms for promoter silencing, such as DNA methylation, it has been demonstrated that the inclusion of EBN1 and OriP sequences into vector constructs may delay the process of promoter silencing (Al-Dosari et al., 2006). Those elements also have been known to play an important role during viral infection in retention, replication, nuclear localization, binding to the nuclear matrix of the target cell, and tran-scriptional up-regulation (Cui et al., 2001). Nevertheless, the mechanism of promoter inactivation remains to be poorly understood. The development of the vectors, which was capable of expressing high levels of transgene products, remains as an occasional finding. A thorough investigation on the mechanisms underlying episomal gene expression would be important for successful development of gene therapy.

Dose-dependent response for AAV gene transfer in other tissue has been previously reported (Klein et al., 2002), and the wide range of doses has established minimal doses for transgene expression $\left(10^{7}\right.$ particles). There is a shift toward lower potency for bicistronic vectors. The present study determined the dose dependency and minimum effective doses for AAV1 gene transfer using CAG promoter for the cochlear cells.

The direct measurement of transgene expression level in individual cells of cochlea in vivo is an important evaluation for cochlear gene therapy. Nevertheless, quantifying the number of cells transduced remains an important additional issue and a combination of protein expression and cell transduction efficiency may permit us to estimate the amount of gene product per cell under appropriate conditions. The gene transduction of the cochlear inner hair cells requires efficient promoter systems, which ensure potent and stable expression of exogenous genes. In this study, we demonstrated that the CAG and CMV promoters showed stable and efficient activity in the cochlear cells, the Myo promoter was specific for gene transfer in the inner hair cells. The stable and efficient promoter activities might be necessary for cochlear gene therapy strategies. From the data obtained by this study, it might be able to extend our knowledge both in the basic and clinical gene research fields.

\section{Acknowledgement}

This study was supported in part by Research Grants from the Ministry of Education, Culture, Sports, Science and Technology, the Ministry of Health, Labor and Welfare, and the Vehicle Racing Commemorative Foundation. 


\section{References}

Al-Dosari M, Zhang G, Knapp JE, Liu D. Evaluation of viral and mammalian promoters for driving transgene expression in mouse liver. Biochem Biophys Res Commun 2006;339:673-8

Boeda B, Weil D, Petit C. A specific promoter of the sensory cells of the inner ear defined by transgenesis. Hum Mol Genet 2001;10:1581-9

Chen ZY, Yant SR, He CY, Meuse L, Shen S, Kay MA. Linear DNAs concatemerize in vivo and result in sustained transgene expression in mouse liver. Mol Ther 2001;3:403-10

Chung S, Andersson T, Sonntag KC, Bjorklund L, Isacson O, Kim KS. Analysis of different promoter systems for efficient transgene expression in mouse embryonic stem cell lines. Stem Cells 2002;20:139-45

Cui FD, Kishida T, Ohashi S, Asada H, Yasutomi K, Satoh E, Kubo T, Fushiki S, Imanishi J, Mazda O. Highly efficient gene transfer into murine liver achieved by intravenous administration of naked Epstein-Barr virus (EBV)-based plasmid vectors. Gene Ther 2001;8:1508-13

Dazert S, Aletsee C, Brors D, Gravel C, Sendtner M, Ryan A. In vivo adenoviral transduction of the neonatal rat cochlea and middle ear. Hear Res 2001;151:30-40

Gill DR, Smyth SE, Goddard CA, Pringle IA, Higgins CF, Colledge $\mathrm{WH}$, Hyde SC. Increased persistence of lung gene expression using plasmids containing the ubiquitin $\mathrm{C}$ or elongation factor 1alpha promoter. Gene Ther 2001;8:1539-46

Hartikka J, Sawdey M, Cornefert-Jensen F, Margalith M, Barnhart K, Nolasco M, Vahlsing HL, Meek J, Marquet M, Hobart P, Norman J, Manthorpe M. An improved plasmid DNA expression vector for direct injection into skeletal muscle. Hum Gene Ther 1996;7:1205-17

Kawamoto K, Oh SH, Kanzaki S, Brown N, Raphael Y. The functional and structural outcome of inner ear gene transfer via the vestibular and cochlear fluids in mice. Mol Ther 2001:4:575-85

Klein RL, Hamby ME, Gong Y, Hirko AC, Wang S, Hughes JA, King MA, Meyer EM. Dose and promoter effects of adeno-associated viral vector for green fluorescent protein expression in the rat brain. Exp Neurol 2002;176: 66-74

Li Duan M, Bordet T, Mezzina M, Kahn A, Ulfendahl M. Adenoviral and adeno-associated viral vector mediated gene transfer in the guinea pig cochlea. Neuroreport 2002;13: $1295-9$

Liu BH, Wang $X$, Ma YX, Wang S. CMV enhancer/human PDGF-beta promoter for neuron-specific transgene expression. Gene Ther 2004;11:52-60
Liu Y, Okada T, Sheykholeslami K, Shimazaki K, Nomoto T, Muramatsu SI, Kanazawa T, Takeuchi K, Ajalli R, Mizukami H, Kume A, Ichimura K, Ozawa K. Specific and efficient transduction of cochlear inner hair cells with recombinant adeno-associated virus type 3 vector. Mol Ther 2005;12:725-733

Luebke AE, Foster PK, Muller CD, Peel AL. Cochlear function and transgene expression in the guinea pig cochlea, using adenovirus- and adeno-associated virus-directed gene transfer. Hum Gene Ther 2001;12:773-81

Matsushita T, Elliger S, Elliger C, Podsakoff G, Villarreal L, Kurtzman GJ, Iwaki Y, Colosi P. Adeno-associated virus vectors can be efficiently produced without helper virus. Gene Ther 1998;5:938-45

Mochizuki S, Mizukami H, Kume A, Muramatsu S, Takeuchi K, Matsushita T, Okada T, Kobayashi E, Hoshika A, Ozawa K. Adeno-associated virus (AAV) vector-mediated liver- and muscle-directed transgene expression using various kinds of promoters and serotypes. Gene ther Mol Biol 2004;8:9-18

Nomoto T, Okada T, Shimazaki K, Mizukami H, Matsushita T, Hanazono Y, Kume A, Katsura K, Katayama Y, Ozawa K. Distinct patterns of gene transfer to gerbil hippocampus with recombinant adeno-associated virus type 2 and 5 . Neurosci Lett 2003;340:153-7

Ogasawara Y, Urabe M, Ozawa K. The use of heterologous promoters for adeno-associated virus (AAV) protein expression in AAV vector production. Microbiol Immunol 1998;42:177-85

Okada T, Nomoto T, Shimazaki K, Lijun W, Lu Y, Matsushita T, Mizukami H, Urabe M, Hanazono Y, Kume A, Muramatsu S, Nakano I, Ozawa K. Adeno-associated virus vectors for gene transfer to the brain. Methods 2002;28:237-47

Shevtsova Z, Malik JM, Michel U, Bahr M, Kugler S. Promoters and serotypes: targeting of adeno-associated virus vectors for gene transfer in the rat central nervous system in vitro and in vivo. Exp Physiol 2005;90:53-9

Veldwijk MR, Topaly J, Laufs S, Hengge UR, Wenz F, Zeller WJ, Fruehauf S. Development and optimization of a real-time quantitative PCR-based method for the titration of AAV-2 vector stocks. Mol Ther 2002;6:272-8

Xu L, Daly T, Gao C, Flotte TR, Song S, Byrne BJ, Sands MS, Parker Ponder K. CMV-beta-actin promoter directs higher expression from an adeno-associated viral vector in the liver than the cytomegalovirus or elongation factor 1 alpha promoter and results in therapeutic levels of human factor $X$ in mice. Hum Gene Ther 2001;12:563-73

Yamasoba T, Suzuki M, Kondo K. Transgene expression in mature guinea pig cochlear cells in vitro. Neurosci Lett 2002;335:13-6 